\title{
AUXILIARY PRINCIPLE FOR GENERALIZED NONLINEAR VARIATIONAL-LIKE INEQUALITIES
}

\author{
ZEQING LIU, HAIYAN GAO, SHIN MIN KANG, AND SOO HAK SHIM
}

Received 27 April 2005; Revised 23 March 2006; Accepted 25 April 2006

We introduce and study a new class of generalized nonlinear variational-like inequalities and prove an existence theorem of solutions for this kind of generalized nonlinear variational-like inequalities. By using the auxiliary principle technique, we construct a new iterative scheme for solving the class of the generalized nonlinear variational-like inequalities. The convergence of the sequence generated by the iterative algorithm is also discussed. Our results extend and unify the corresponding results due to Ding, Liu, Ume, Kang, Yao, and others.

Copyright @ 2006 Hindawi Publishing Corporation. All rights reserved.

\section{Introduction}

Variational inequality theory has become a very effective and powerful tool for studying a wide range of problems arising in many diverse fields of pure and applied sciences. It is well known that one of the most important problems in variational inequality theory is the development of efficient and implementable iterative algorithms for solving various classes of variational inequalities and variational inclusions. In [3-38] there are a lot of iterative algorithms for finding the approximate solutions of various variational inequalities. Glowinski et al. [8] had developed the auxiliary principle technique. By using the auxiliary principle technique, Ding [3,4], Ding and Tan [5], and Ding and Yao [6], Liu et al. [21, 28], Zeng et al. [37], Zeng et al. [38], and others suggested several iterative algorithms to compute approximate solutions for some classes of general nonlinear mixed variational inequalities and variational-like inequalities in reflexive Banach spaces.

Motivated and inspired by the research work in [3-38], in this paper, we introduce and study a new class of generalized nonlinear variational-like inequalities and prove an existence theorem of solutions for this kind of generalized nonlinear variational-like inequalities. By applying the result due to Chang $[1,2]$ and the auxiliary principle technique, we suggest a new iterative scheme for solving the class of generalized nonlinear variational-like inequalities. The convergence of the sequence generated by the iterative

Hindawi Publishing Corporation

International Journal of Mathematics and Mathematical Sciences

Volume 2006, Article ID 95723, Pages 1-11

DOI 10.1155/IJMMS/2006/95723 
2 Generalized nonlinear variational-like inequalities

algorithm is also discussed. Our results extend and unify the corresponding results due to Ding [3], Liu et al. [21], Yao [36], and others.

\section{Preliminaries}

Throughout this paper, we assume that $H$ is a real Hilbert space with dual space $H^{*}$ and that $\langle u, v\rangle$ is the dual pairing between $u \in H$ and $v \in H^{*}$. Let $K$ be a nonempty closed convex subset of $H$, and let $A, B, C: K \rightarrow H, N: H \times H \times H \rightarrow H$, and $\eta: K \times K \rightarrow H^{*}$ be mappings. Suppose that $a: K \times K \rightarrow(-\infty, \infty)$ is a coercive continuous bilinear form, that is, there exist positive constants $c, d>0$ such that

(C1) $a(v, v) \geq c\|v\|^{2}$ for all $v \in K$;

(C2) $|a(u, v)| \leq d\|u\| \cdot\|v\|$ for all $u, v \in K$. It follows from (C1) and (C2) that $c \leq d$. Let $b: K \times K \rightarrow(-\infty,+\infty)$ be nondifferentiable and satisfy the following conditions:

(C3) $b$ is linear in the first argument;

(C4) $b$ is convex in the second argument;

(C5) $b$ is bounded, that is, there exists a constant $l>0$ satisfying

$$
|b(u, v)| \leq l\|u\| \cdot\|v\| \quad \forall u, v \in K
$$

(C6) $b(u, v)-b(u, w) \leq b(u, v-w)$ for all $u, v, w \in K$.

Now we consider the following generalized nonlinear variational-like inequality.

For given $f \in H$, find $u \in K$ such that

$$
\langle N(A u, B u, C u)-f, \eta(v, u)\rangle+a(u, v-u) \geq b(u, u)-b(u, v) \quad \forall v \in K .
$$

Special cases. If $N(A u, B u, C u)=A u-B u, f=0$, and $b(u, v)=\varphi(v)$ for all $u, v \in K$, where $\varphi: H \rightarrow(-\infty,+\infty)$ is a functional, then the generalized nonlinear variational-like inequality (2.2) is equivalent to finding $u \in K$ such that

$$
\langle A u-B u, \eta(v, u)\rangle \geq \varphi(u)-\varphi(v) \quad \forall v \in K
$$

which was introduced and studied by Ding [3].

If $N(A u, B u, C u)=A u-B u, f=0, b(u, v)=\varphi(v)$, and $\eta(u, v)=g u-g v$ for all $u, v \in K$, where $g: K \rightarrow H^{*}$ is a mapping, then the generalized nonlinear variational-like inequality (2.2) is equivalent to finding $u \in K$ such that

$$
\langle A u-B u, g v-g u\rangle \geq \varphi(u)-\varphi(v) \quad \forall v \in K,
$$

which was studied by Yao [36].

Definition 2.1. Let $A, B: K \rightarrow H, N: H \times H \times H \rightarrow H$, and $\eta: K \times K \rightarrow H^{*}$ be mappings.

(1) $A$ is said to be Lipschitz continuous with constant $r$ if there exists a constant $r>0$ such that

$$
\|A x-A y\| \leq r\|x-y\| \quad \forall x, y \in K
$$


(2) $N$ is said to be $\eta$-strongly monotone with constant $s$ with respect to $A$ in the first argument if there exists a constant $s>0$ such that

$$
\langle N(A x, u, v)-N(A y, u, v), \eta(x, y)\rangle \geq s\|x-y\|^{2} \quad \forall x, y \in K, \forall u, v \in H .
$$

(3) $N$ is said to be $\eta$-monotone with respect to $A$ in the second argument if

$$
\langle N(u, A x, v)-N(v, A y, v), \eta(x, y)\rangle \geq 0 \quad \forall x, y \in K, \forall u, v \in H .
$$

(4) $N$ is said to be Lipschitz continuous with constant $t$ in the third argument if there exists a constant $t>0$ such that

$$
\|N(u, v, x)-N(u, v, y)\| \leq t\|x-y\| \quad \forall x, y, u, v \in H .
$$

(5) $N$ is said to be $\eta$-hemicontinuous with respect to $A$ and $B$ in the first and second arguments if for any $x, y, z \in K$, the mapping $g:[0,1] \rightarrow(-\infty, \infty)$ defined by $g(t)=$ $\langle N(A(t x+(1-t) y), B(t x+(1-t) y), z), \eta(x, y)\rangle$ is continuous at $0^{+}$.

(6) $\eta$ is said to be Lipschitz continuous with constant $s$ if there exists a constant $s>0$ such that

$$
\|\eta(x, y)\| \leq s\|x-y\| \quad \forall x, y \in K \text {. }
$$

(7) $\eta$ is said to be strongly monotone with constant $t$ if there exists a constant $t>0$ such that

$$
\langle x-y, \eta(x, y)\rangle \geq t\|x-y\|^{2} \quad \forall x, y \in K
$$

Lemma $2.2[1,2]$. Let $X$ be a nonempty closed convex subset of a Hausdorff linear topological space $E$, and let $\phi, \psi: X \times X \rightarrow R$ be mappings satisfying the following conditions:

(a) $\psi(x, y) \leq \phi(x, y)$ for all $x, y \in X$, and $\psi(x, x) \geq 0$ for all $x \in X$;

(b) for each $x \in X, \phi(x, \cdot)$ is upper semicontinuous on $X$;

(c) for each $y \in X$, the set $\{x \in X: \psi(x, y)<0\}$ is a convex set;

(d) there exists a nonempty compact set $K \subset X$ and $x_{0} \in K$ such that $\psi\left(x_{0}, y\right)<0$ for all $y \in X \backslash K$.

Then there exists $\hat{y} \in K$ such that $\phi(x, \hat{y}) \geq 0$ for all $x \in X$.

\section{Auxiliary problem and algorithm}

Now we consider the following auxiliary problem with respect to the generalized nonlinear variational-like inequality (2.2). For any given $u \in K$, find $\widehat{w} \in K$ such that

$$
\begin{aligned}
\langle\widehat{w}, \eta(v, \widehat{w})\rangle \geq & \langle u, \eta(v, \widehat{w})\rangle-\rho\langle N(A \widehat{w}, B \widehat{w}, C u)-f, \eta(v, \widehat{w})\rangle \\
& -\rho a(\widehat{w}, v-\widehat{w})-\rho b(u, v)+\rho b(u, \widehat{w}) \quad \forall v \in K,
\end{aligned}
$$

where $\rho>0$ is a constant. 
Theorem 3.1. Let $K$ be a nonempty closed convex subset of $H$ and $f \in H$. Suppose that $a$ : $K \times K \rightarrow(-\infty, \infty)$ satisfies (C1) and (C2), $b: K \times K \rightarrow(-\infty, \infty)$ satisfies (C3)-(C6), and $A, B, C: K \rightarrow H$ and $N: H \times H \times H \rightarrow H$ are mappings such that $N$ is $\eta$-hemicontinuous with respect to $A$ and $B$ in the first and second arguments. Let $\eta: K \times K \rightarrow H^{*}$ be Lipschitz continuous with constant $\delta$ and strongly monotone with constant $\tau$, for each $y \in$ $K$, let $\eta(\cdot, y)$ be continuous and $\eta(y, x)=-\eta(x, y)$ for all $x, y \in K$. Assume that $N$ is $\eta$ strongly monotone with constant $\alpha$ with respect to $A$ in the first argument and $\eta$-monotone with respect to $B$ in the second argument. If for given $x, y, z \in H$ and $v \in K$, the mapping $\langle N(x, y, z), \eta(v, \cdot)\rangle$ is concave and upper semicontinuous, then the auxiliary problem (3.1) has a unique solution in $K$.

Proof. Let $u$ be in $K$. Define the functionals $\phi$ and $\psi: K \times K \rightarrow R$ by

$$
\begin{aligned}
\phi(v, w)= & \langle v, \eta(v, w)\rangle-\langle u, \eta(v, w)\rangle+\rho\langle N(A v, B v, C u)-f, \eta(v, w)\rangle \\
& +\rho a(v, v-w)-\rho b(u, w)+\rho b(u, v) \\
\psi(v, w)= & \langle w, \eta(v, w)\rangle-\langle u, \eta(v, w)\rangle+\rho\langle N(A w, B w, C u)-f, \eta(v, w)\rangle \\
& +\rho a(w, v-w)-\rho b(u, w)+\rho b(u, v)
\end{aligned}
$$

for all $v, w \in K$.

We check that the functionals $\phi$ and $\psi$ satisfy all the conditions of Lemma 2.2 in the weak topology. It is easy to see for all $v, w \in K$,

$$
\begin{aligned}
\phi(v, w) & -\psi(v, w) \\
= & \langle v-w, \eta(v, w)\rangle+\rho\langle N(A v, B v, C u)-N(A w, B v, C u), \eta(v, w)\rangle \\
& +\rho\langle N(A w, B v, C u)-N(A w, B w, C u), \eta(v, w)\rangle+\rho a(v-w, v-w) \\
\geq & {[\tau+\rho(\alpha+c)]\|v-w\|^{2} \geq 0 }
\end{aligned}
$$

which implies that $\phi$ and $\psi$ satisfy the condition (1) of Lemma 2.2. Since $a$ is a coercive continuous bilinear form, it follows that $a(v, v-w)$ is weakly upper semicontinuous with respect to $w$. Note that $b$ is convex and lower semicontinuous in the second argument and for given $x, y, z \in H, v \in K$, the mapping $\langle N(x, y, z), \eta(v, \cdot)\rangle$ is concave and upper semicontinuous. Therefore $\phi(v, \cdot)$ is weakly upper semicontinuous in the second argument and the set $\{v \in K: \psi(v, w)<0\}$ is convex for each $w \in K$. That is, the conditions (2) and (3) of Lemma 2.2 hold. Let $\bar{v} \in K$. Put

$$
\begin{gathered}
L=[\tau+\rho(\alpha+c)]^{-1}[\delta\|u-\bar{v}\|+\rho d\|\bar{v}\|+\rho \delta\|N(A \bar{v}, B \bar{v}, C u)-f\|+\rho l\|u\|], \\
M=\{w \in K:\|w-\bar{v}\| \leq L\} .
\end{gathered}
$$


Clearly, $M$ is a weakly compact subset of $K$ and for any $w \in K \backslash M$,

$$
\begin{aligned}
\psi(\bar{v}, w)= & \langle w, \eta(\bar{v}, w)\rangle-\langle u, \eta(\bar{v}, w)\rangle \\
& +\rho\langle N(A w, B w, C u)-f, \eta(\bar{v}, w)\rangle \\
& +\rho a(w, \bar{v}-w)-\rho b(u, w)+\rho b(u, \bar{v}) \\
\leq & -\langle w-\bar{v}, \eta(w, \bar{v})\rangle+\langle u-\bar{v}, \eta(w, \bar{v})\rangle \\
& -\rho\langle N(A w, B w, C u)-N(A \bar{v}, B w, C u), \eta(w, \bar{v})\rangle \\
& -\rho\langle N(A \bar{v}, B w, C u)-N(A \bar{v}, B \bar{v}, C u), \eta(w, \bar{v})\rangle \\
& -\rho\langle N(A \bar{v}, B \bar{v}, C u)-f, \eta(w, \bar{v})\rangle \\
& -\rho a(w-\bar{v}, w-\bar{v})-\rho a(\bar{v}, w-\bar{v})+\rho b(u, \bar{v}-w) \\
\leq & -\|w-\bar{v}\|\{[\tau+\rho(\alpha+c)]\|w-\bar{v}\|-\delta\|u-\bar{v}\| \\
& \quad-\rho d\|\bar{v}\|-\rho \delta\|N(A \bar{v}, B \bar{v}, C u)-f\|-\rho l\|u\|\}<0,
\end{aligned}
$$

which means that the condition (4) of Lemma 2.2 holds. Thus Lemma 2.2 ensures that there exists $\widehat{w} \in K$ such that $\phi(v, \widehat{w}) \geq 0$ for all $v \in K$, that is,

$$
\begin{aligned}
\langle v, \eta(v, \widehat{w})\rangle \geq & \langle u, \eta(v, \widehat{w})\rangle-\rho\langle N(A v, B v, C u)-f, \eta(v, \widehat{w})\rangle \\
& -\rho a(v, v-\widehat{w})-\rho b(u, v)+\rho b(u, \widehat{w}) \quad \forall v \in K .
\end{aligned}
$$

Let $t$ be in $(0,1]$ and let $v$ be in $K$. Replacing $v$ by $v_{t}=t v+(1-t) \widehat{w}$ in $(3.6)$, we see that

$$
\begin{aligned}
\left\langle v_{t}, \eta\left(v_{t}, \widehat{w}\right)\right\rangle \geq & \left\langle u, \eta\left(v_{t}, \widehat{w}\right)\right\rangle-\rho\left\langle N\left(A v_{t}, B v_{t}, C u\right)-f, \eta\left(v_{t}, \widehat{w}\right)\right\rangle \\
& -\rho a\left(v_{t}, v_{t}-\widehat{w}\right)-\rho b\left(u, v_{t}\right)+\rho b(u, \widehat{w}) \quad \forall v \in K .
\end{aligned}
$$

Notice that $b$ is convex in the second argument and $\langle N(x, y, z), \eta(\nu, \cdot)\rangle$ is concave and upper semicontinuous. From (C6) and (3.7) we infer that

$$
\begin{aligned}
t\left[\left\langle v_{t}, \eta(v, \widehat{w})\right\rangle\right] \geq t[ & \langle u, \eta(v, \widehat{w})\rangle-\rho\left\langle N\left(A v_{t}, B v_{t}, C u\right)-f, \eta(v, \widehat{w})\right\rangle \\
& \left.-\rho a\left(v_{t}, v-\widehat{w}\right)-\rho b(u, v)+\rho b(u, \widehat{w})\right] \quad \forall v \in K,
\end{aligned}
$$

which implies that

$$
\begin{aligned}
\left\langle v_{t}, \eta(v, \widehat{w})\right\rangle \geq & \langle u, \eta(v, \widehat{w})\rangle-\rho\left\langle N\left(A v_{t}, B v_{t}, C u\right)-f, \eta(v, \widehat{w})\right\rangle \\
& -\rho a\left(v_{t}, v-\widehat{w}\right)-\rho b(u, v)+\rho b(u, \widehat{w}) \quad \forall v \in K .
\end{aligned}
$$


6 Generalized nonlinear variational-like inequalities

Letting $t \rightarrow 0^{+}$in the above inequality, we conclude that

$$
\begin{aligned}
\langle\widehat{w}, \eta(v, \widehat{w})\rangle \geq & \langle u, \eta(v, \widehat{w})\rangle-\rho\langle N(A \widehat{w}, B \widehat{w}, C u)-f, \eta(v, \widehat{w})\rangle \\
& -\rho a(\widehat{w}, v-\widehat{w})-\rho b(u, v)+\rho b(u, \widehat{w}) \quad \forall v \in K .
\end{aligned}
$$

That is, $\widehat{w}$ is a solution of (3.1). Now we prove the uniqueness. For any two solutions $w_{1}, w_{2} \in K$ of (3.1) with respect to $u$, we know that

$$
\begin{aligned}
\left\langle w_{1}, \eta\left(v, w_{1}\right)\right\rangle \geq & \left\langle u, \eta\left(v, w_{1}\right)\right\rangle-\rho\left\langle N\left(A w_{1}, B w_{1}, C u\right)-f, \eta\left(v, w_{1}\right)\right\rangle \\
& -\rho a\left(w_{1}, v-w_{1}\right)-\rho b(u, v)+\rho b\left(u, w_{1}\right), \\
\left\langle w_{2}, \eta\left(v, w_{2}\right)\right\rangle \geq & \left\langle u, \eta\left(v, w_{2}\right)\right\rangle-\rho\left\langle N\left(A w_{2}, B w_{2}, C u\right)-f, \eta\left(v, w_{2}\right)\right\rangle \\
& -\rho a\left(w_{2}, v-w_{2}\right)-\rho b(u, v)+\rho b\left(u, w_{2}\right)
\end{aligned}
$$

for all $v \in K$. Taking $v=w_{2}$ in (3.11) and $v=w_{1}$ in (3.12), we get that

$$
\begin{aligned}
\left\langle w_{1}, \eta\left(w_{2}, w_{1}\right)\right\rangle \geq & \left\langle u, \eta\left(w_{2}, w_{1}\right)\right\rangle-\rho\left\langle N\left(A w_{1}, B w_{1}, C u\right)-f, \eta\left(w_{2}, w_{1}\right)\right\rangle \\
& -\rho a\left(w_{1}, w_{2}-w_{1}\right)-\rho b\left(u, w_{2}\right)+\rho b\left(u, w_{1}\right), \\
\left\langle w_{2}, \eta\left(w_{1}, w_{2}\right)\right\rangle \geq & \left\langle u, \eta\left(w_{1}, w_{2}\right)\right\rangle-\rho\left\langle N\left(A w_{2}, B w_{2}, C u\right)-f, \eta\left(w_{1}, w_{2}\right)\right\rangle \\
& -\rho a\left(w_{2}, w_{1}-w_{2}\right)-\rho b\left(u, w_{1}\right)+\rho b\left(u, w_{2}\right) .
\end{aligned}
$$

Adding these inequalities, we deduce that

$$
\begin{aligned}
\tau\left\|w_{1}-w_{2}\right\|^{2} \leq & -\rho\left\langle N\left(A w_{1}, B w_{1}, C u\right)-N\left(A w_{2}, B w_{1}, C u\right), \eta\left(w_{1}, w_{2}\right)\right\rangle \\
& -\rho\left\langle N\left(A w_{2}, B w_{1}, C u\right)-N\left(A w_{2}, B w_{2}, C u\right), \eta\left(w_{1}, w_{2}\right)\right\rangle \\
& -\rho a\left(w_{1}-w_{2}, w_{1}-w_{2}\right) \\
\leq & -\rho(\alpha+c)\left\|w_{1}-w_{2}\right\|^{2},
\end{aligned}
$$

which yields $w_{1}=w_{2}$. That is, $\widehat{w}$ is the unique solution of (3.1). This completes the proof.

By Theorem 3.1, we suggest the following algorithms for solving the generalized nonlinear variational-like inequality (2.2).

Algorithm 3.2. Suppose that $a: K \times K \rightarrow(-\infty, \infty)$ satisfies (C1), (C2), $b: K \times K \rightarrow$ $(-\infty, \infty)$ satisfies (C3)-(C6), and $A, B, C: K \rightarrow H, N: H \times H \times H \rightarrow H$ and $\eta: K \times K \rightarrow$ $H^{*}$ are mappings. For given $f \in H$ and $u_{0} \in K$, compute the sequence $\left\{u_{n}\right\}_{n \geq 0} \subset K$ by the following iterative scheme:

$$
\begin{aligned}
\left\langle u_{n+1}, \eta\left(v, u_{n+1}\right)\right\rangle \geq & \left\langle u_{n}, \eta\left(v, u_{n+1}\right)\right\rangle-\rho\left\langle N\left(A u_{n+1}, B u_{n+1}, C u_{n}\right)-f, \eta\left(v, u_{n+1}\right)\right\rangle \\
& -\rho a\left(u_{n+1}, v-u_{n+1}\right)-\rho b\left(u_{n}, v\right)+\rho b\left(u_{n}, u_{n+1}\right)+\left\langle e_{n}, \eta\left(v, u_{n+1}\right)\right\rangle
\end{aligned}
$$

for all $v \in K$ and $n \geq 0$, where $\left\{e_{n}\right\}_{n \geq 0} \subset H$ and $\rho>0$ is a constant. 


\section{Existence and convergence}

In this section, we prove the existence of solution for the generalized nonlinear variational-like inequality (2.2) and discuss the convergence of the sequence generated by Algorithm 3.2.

Theorem 4.1. Let $a, b, A, B, N, \eta$ be as in Theorem 3.1. Let $C: K \rightarrow H$ be Lipschitz continuous with constant $\xi$. Assume that $N$ is Lipschitz continuous with constant $\sigma$ in the third argument and strongly monotone with constant $\beta$ with respect to $C$ in the third argument and

$$
\sigma \xi \geq \beta, \quad k=\frac{l-\alpha-c}{\delta}, \quad p=\frac{\tau}{\delta}, \quad \lim _{n \rightarrow \infty}\left\|e_{n}\right\|=0
$$

If there exist a constant $\rho$ satisfying

$$
0<\rho<\frac{\tau}{l-\alpha-c}
$$

and one of the following conditions:

$$
\begin{gathered}
\left|\rho-\frac{\beta-p k}{\sigma^{2} \xi^{2}-k^{2}}\right|<\frac{\sqrt{(\beta-p k)^{2}-\left(\sigma^{2} \xi^{2}-k^{2}\right)\left(1-p^{2}\right)}}{\sigma^{2} \xi^{2}-k^{2}}, \\
\sigma \xi>k, \quad|\beta-p k|>\sqrt{\left(\sigma^{2} \xi^{2}-k^{2}\right)\left(1-p^{2}\right)}, \\
\left|\rho-\frac{p k-\beta}{k^{2}-\sigma^{2} \xi^{2}}\right|>\frac{\sqrt{(\beta-p k)^{2}+\left(k^{2}-\sigma^{2} \xi^{2}\right)\left(1-p^{2}\right)}}{k^{2}-\sigma^{2} \xi^{2}}, \quad \sigma \xi<k,
\end{gathered}
$$

then the iterative sequence $\left\{u_{n}\right\}_{n \geq 0}$ generated by Algorithm 3.2 converges strongly to some $u \in K$ and $u$ is a solution of the generalized nonlinear variational-like inequality (2.2).

Proof. It follows from the proof of Theorem 3.1 that there exists a mapping $G: K \rightarrow K$ satisfying $G(u)=w$, where $w$ is the unique solution of (3.1) for each $u \in K$. Next we show that $G$ is a contraction mapping. Let $u_{1}$ and $u_{2}$ be arbitrary elements in $K$. Using (3.1), we see that

$$
\begin{aligned}
\left\langle G u_{1}, \eta\left(v, G u_{1}\right)\right\rangle \geq & \left\langle u_{1}, \eta\left(v, G u_{1}\right)\right\rangle-\rho\left\langle N\left(A\left(G u_{1}\right), B\left(G u_{1}\right), C u_{1}\right)-f, \eta\left(v, G u_{1}\right)\right\rangle \\
& -\rho a\left(G u_{1}, v-G u_{1}\right)-\rho b\left(u_{1}, v\right)+\rho b\left(u_{1}, G u_{1}\right), \\
\left\langle G u_{2}, \eta\left(v, G u_{2}\right)\right\rangle \geq & \left\langle u_{2}, \eta\left(v, G u_{2}\right)\right\rangle-\rho\left\langle N\left(A\left(G u_{2}\right), B\left(G u_{2}\right), C u_{2}\right)-f, \eta\left(v, G u_{2}\right)\right\rangle \\
& -\rho a\left(G u_{2}, v-G u_{2}\right)-\rho b\left(u_{2}, v\right)+\rho b\left(u_{2}, G u_{2}\right)
\end{aligned}
$$


8 Generalized nonlinear variational-like inequalities

for all $v \in K$. Letting $v=G u_{2}$ in (4.4) and $v=G u_{1}$ in (4.5), and adding these inequalities, we arrive at

$$
\begin{aligned}
\tau\left\|G u_{1}-G u_{2}\right\|^{2} \leq & \left\langle G u_{1}-G u_{2}, \eta\left(G u_{1}, G u_{2}\right)\right\rangle \\
\leq & \left\langle u_{1}-u_{2}-\rho\left(N\left(A\left(G u_{2}\right), B\left(G u_{2}\right), C u_{1}\right)\right.\right. \\
& \left.\left.-N\left(A\left(G u_{2}\right), B\left(G u_{2}\right), C u_{2}\right)\right), \eta\left(G u_{1}, G u_{2}\right)\right\rangle \\
& -\rho\left\langle N\left(A\left(G u_{1}\right), B\left(G u_{1}\right), C u_{1}\right)\right. \\
& \left.-N\left(A\left(G u_{2}\right), B\left(G u_{1}\right), C u_{1}\right), \eta\left(G u_{1}, G u_{2}\right)\right\rangle \\
& -\rho\left\langle N\left(A\left(G u_{2}\right), B\left(G u_{1}\right), C u_{1}\right)\right. \\
& \left.-N\left(A\left(G u_{2}\right), B\left(G u_{2}\right), C u_{1}\right), \eta\left(G u_{1}, G u_{2}\right)\right\rangle \\
& -\rho a\left(G u_{1}-G u_{2}, G u_{1}-G u_{2}\right)+\rho b\left(u_{1}-u_{2}, G u_{2}-G u_{1}\right) \\
\leq & \left.\delta \sqrt{1-2 \rho \beta+(\rho \sigma \xi)^{2}}+\rho l\right]\left\|u_{1}-u_{2}\left|\left\||| G u_{1}-G u_{2}\right\|\right.\right. \\
& -\rho(\alpha+c)\left\|G u_{1}-G u_{2}\right\|^{2},
\end{aligned}
$$

that is,

$$
\left\|G u_{1}-G u_{2}\right\| \leq \theta\left\|u_{1}-u_{2}\right\|
$$

where

$$
\theta=\frac{\delta \sqrt{1-2 \rho \beta+(\rho \sigma \xi)^{2}}+\rho l}{\tau+\rho(\alpha+c)}<1
$$

by (4.2) and one of (4.3). Therefore, $G: K \rightarrow K$ is a contraction mapping and has a unique fixed point $u \in K$. It follows from (3.1) that

$$
\begin{aligned}
\langle u, \eta(v, u)\rangle \geq & \langle u, \eta(v, u)\rangle-\rho\langle N(A u, B u, C u)-f, \eta(v, u)\rangle \\
& -\rho a(u, v-u)-\rho b(u, v)+\rho b(u, u) \quad \forall v \in K,
\end{aligned}
$$

which implies that

$$
\langle N(A u, B u, C u)-f, \eta(v, u)\rangle+a(u, v-u) \geq b(u, u)-b(u, v) \quad \forall v \in K,
$$

that is, $u$ is a solution of the generalized nonlinear variational-like inequality (2.2). 
Next, we consider the convergence of the iterative sequence generated by Algorithm 3.2. Taking $v=u_{n+1}$ in (4.9) and $v=u$ in (3.15), and adding these inequalities, we have

$$
\begin{aligned}
\tau\left\|u_{n+1}-u\right\|^{2} \leq & \left\langle u_{n+1}-u, \eta\left(u_{n+1}, u\right)\right\rangle \\
\leq & \left\langle u_{n}-u-\rho\left(N\left(A u, B u, C u_{n}\right)-N(A u, B u, C u)\right), \eta\left(u_{n+1}, u\right)\right\rangle \\
& -\rho\left\langle N\left(A u_{n+1}, B u_{n+1}, C u_{n}\right)-N\left(A u, B u_{n+1}, C u\right), \eta\left(u_{n+1}, u\right)\right\rangle \\
& -\rho\left\langle N\left(A u, B u_{n+1}, C u_{n}\right)-N\left(A u, B u, C u_{n}\right), \eta\left(u_{n+1}, u\right)\right\rangle \\
& -\rho a\left(u_{n+1}-u, u_{n+1}-u\right)+\rho b\left(u_{n}-u, u-u_{n+1}\right)+\left\langle e_{n}, \eta\left(u_{n+1}, u\right)\right\rangle \\
\leq & {\left[\delta \sqrt{1-2 \rho \beta+(\rho \sigma \xi)^{2}}+\rho l\right]\left\|u_{n}-u\right\|\left\|u_{n+1}-u\right\| } \\
& -\rho(\alpha+c)\left\|u_{n+1}-u\right\|^{2}+\left\|e_{n}\right\|\left\|u_{n+1}-u\right\|
\end{aligned}
$$

for all $n \geq 1$. That is,

$$
\left\|u_{n+1}-u\right\| \leq \theta\left\|u_{n}-u\right\|+\left\|e_{n}\right\| \rightarrow 0 \text { as } n \rightarrow \infty,
$$

where $\theta$ is defined by (4.8). It follows from (4.1) and (4.12) that the iterative sequence $\left\{u_{n}\right\}_{n \geq 0}$ generated by Algorithm 3.2 converges strongly to $u$. This completes the proof.

\section{Acknowledgments}

This work was supported by the Science Research Foundation of Educational Department of Liaoning Province (2006) and Korea Research Foundation Grant (KRF-2003005-C00013).

\section{References}

[1] S. S. Chang, Variational Inequalitity and Complementarity Theory with Applications, Shanghai Scientific Technology, Shanghai, 1991.

[2] __ On the existence of solutions for a class of quasi-bilinear variational inequalities, Journal of Systems Science and Mathematical Sciences 16 (1996), no. 2, 136-140 (Chinese).

[3] X. P. Ding, Algorithm of solutions for mixed-nonlinear variational-like inequalities in reflexive Banach space, Applied Mathematics and Mechanics 19 (1998), no. 6, 521-529.

[4] __ Existence and algorithm of solutions for nonlinear mixed variational-like inequalities in Banach spaces, Journal of Computational and Applied Mathematics 157 (2003), no. 2, 419-434.

[5] X. P. Ding and K.-K. Tan, A minimax inequality with applications to existence of equilibrium point and fixed point theorems, Colloquium Mathematicum 63 (1992), no. 2, 233-247.

[6] X. P. Ding and J.-C. Yao, Existence and algorithm of solutions for mixed quasi-variational-like inclusions in Banach spaces, Computers \& Mathematics with Applications 49 (2005), no. 5-6, 857-869.

[7] I. Ekeland and R. Temam, Convex Analysis and Variational Problems, North-Holland, Amsterdam, 1976.

[8] R. Glowinski, J.-L. Lions, and R. Trémolières, Numerical Analysis of Variational Inequalities, Studies in Mathematics and Its Applications, vol. 8, North-Holland, Amsterdam, 1981. 
[9] N. J. Huang and C.-X. Deng, Auxiliary principle and iterative algorithms for generalized set-valued strongly nonlinear mixed variational-like inequalities, Journal of Mathematical Analysis and Applications 256 (2001), no. 2, 345-359.

[10] Z. Liu, L. Debnath, S. M. Kang, and J. S. Ume, Completely generalized multivalued nonlinear quasi-variational inclusions, International Journal of Mathematics and Mathematical Sciences 30 (2002), no. 10, 593-604.

[11] _ On the generalized nonlinear quasi-variational inclusions, Acta Mathematica et Informatica Universitatis Ostraviensis 11 (2003), 81-90.

[12] __ Sensitivity analysis for parametric completely generalized nonlinear implicit quasivariational inclusions, Journal of Mathematical Analysis and Applications 277 (2003), no. 1, 142-154.

[13] _ Generalized mixed quasivariational inclusions and generalized mixed resolvent equations for fuzzy mappings, Applied Mathematics and Computation 149 (2004), no. 3, 879-891.

[14] Z. Liu and S. M. Kang, Comments on the papers involving variational and quasivariational inequalities for fuzzy mappings, Mathematical Sciences Research Journal 7 (2003), no. 10, 394-339.

[15] _ Generalized multivalued nonlinear quasi-variational inclusions, Mathematische Nachrichten 253 (2003), no. 1, 45-54.

[16] Convergence and stability of perturbed three-step iterative algorithm for completely generalized nonlinear quasivariational inequalities, Applied Mathematics and Computation 149 (2004), no. 1, 245-258.

[17] Z. Liu, S. M. Kang, and J. S. Ume, On general variational inclusions with noncompact valued mappings, Advances in Nonlinear Variational Inequalities 5 (2002), no. 2, 11-25.

[18]__ Completely generalized multivalued strongly quasivariational inequalities, Publicationes Mathematicae Debrecen 62 (2003), no. 1-2, 187-204.

[19] _ Generalized variational inclusions for fuzzy mappings, Advances in Nonlinear Variational Inequalities 6 (2003), no. 1, 31-40.

[20] The solvability of a class of quasivariational inequalities, Advances in Nonlinear Variational Inequalities 6 (2003), no. 2, 69-78.

[21] Z. Liu, J. S. Ume, and S. M. Kang, General strongly nonlinear quasivariational inequalities with relaxed Lipschitz and relaxed monotone mappings, Journal of Optimization Theory and Applications 114 (2002), no. 3, 639-656.

[22] __ Resolvent equations technique for general variational inclusions, Proceedings of the Japan Academy. Series A. Mathematical Sciences 78 (2002), no. 10, 188-193.

[23] _ Nonlinear variational inequalities on reflexive Banach spaces and topological vector spaces, International Journal of Mathematics and Mathematical Sciences 2003 (2003), no. 4, 199-207.

[24] Completely generalized quasivariational inequalities, Advances in Nonlinear Variational Inequalities 7 (2004), no. 1, 35-46.

[25] Completely generalized quasivariational inequalities, International Journal of Pure and Applied Mathematics 16 (2004), no. 1, 101-112.

[26] General variational inclusions and general resolvent equations, Bulletin of the Korean Mathematical Society 41 (2004), no. 2, 241-256.

[27] Stability of Noor iterations with errors for generalized nonlinear complementarity problems, Acta Mathematica. Academiae Paedagogicae Nyíregyháziensis. New Series 20 (2004), no. 1, 53-61.

[28]_, Generalized nonlinear variational-like inequalities in reflexive Banach spaces, Journal of Optimization Theory and Applications 126 (2005), no. 1, 157-174.

[29] Z. Liu, Li Wang, S. M. Kang, and J. S. Ume, Sensitivity analysis of solutions for generalized parametric nonlinear variational inequalities, International Journal of Pure and Applied Mathematics 6 (2003), no. 1, 77-91. 
[30] P. D. Panagiotopoulos and G. E. Stavroulakis, New types of variational principles based on the notion of quasidifferentiability, Acta Mechanica 94 (1992), no. 3-4, 171-194.

[31] J. Parida and A. Sen, A variational-like inequality for multifunctions with applications, Journal of Mathematical Analysis and Applications 124 (1987), no. 1, 73-81.

[32] A. H. Siddiqi and Q. H. Ansari, Strongly nonlinear quasivariational inequalities, Journal of Mathematical Analysis and Applications 149 (1990), no. 2, 444-450.

[33] _ General strongly nonlinear variational inequalities, Journal of Mathematical Analysis and Applications 166 (1992), no. 2, 386-392.

[34] G. Q. Tian, Generalized quasi-variational-like inequality problem, Mathematics of Operations Research 18 (1993), no. 3, 752-764.

[35] J. C. Yao, The generalized quasi-variational inequality problem with applications, Journal of Mathematical Analysis and Applications 158 (1991), no. 1, 139-160.

[36] _ Existence of generalized variational inequalities, Operations Research Letters 15 (1994), no. $1,35-40$.

[37] L.-C. Zeng, S.-M. Guu, and J.-C. Yao, Iterative algorithm for completely generalized set-valued strongly nonlinear mixed variational-like inequalities, Computers \& Mathematics with Applications 50 (2005), no. 5-6, 935-945.

[38] L. C. Zeng, S. Schaible, and J. C. Yao, Iterative algorithm for generalized set-valued strongly nonlinear mixed variational-like inequalities, Journal of Optimization Theory and Applications $\mathbf{1 2 4}$ (2005), no. 3, 725-738.

Zeqing Liu: Department of Mathematics, Liaoning Normal University, P.O. Box 200, Dalian, Liaoning 116029, China

E-mail address: zeqingliu@dl.cn

Haiyan Gao: Kingbridge Business College, Dongbei University of Finance and Economics, Dalian, Liaoning 116600, China

E-mail address: hygao@163.com

Shin Min Kang: Department of Mathematics and Research Institute of Natural Science,

Gyeongsang National University, Jinju 660-701, South Korea

E-mail address: smkang@nongae.gsnu.ac.kr

Soo Hak Shim: The Research Institute of Natural Science, Gyeongsang National University, Jinju 660-701, South Korea

E-mail address: math@nongae.gsnu.ac.kr 


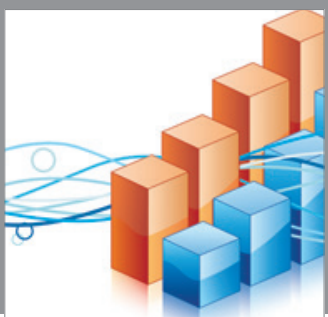

Advances in

Operations Research

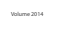

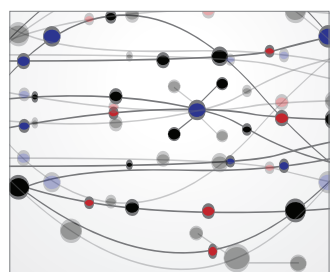

\section{The Scientific} World Journal
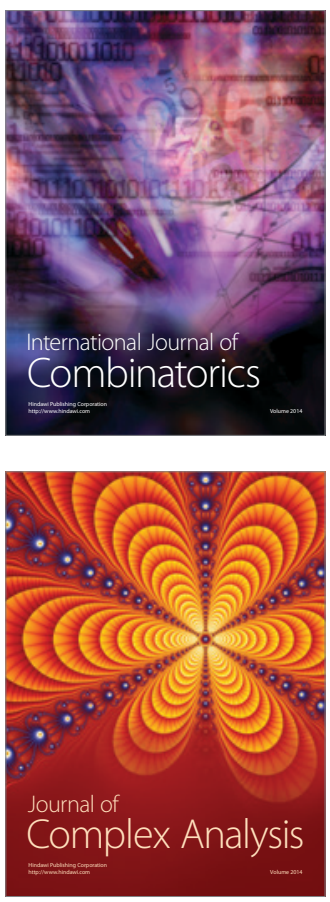

International Journal of

Mathematics and

Mathematical

Sciences
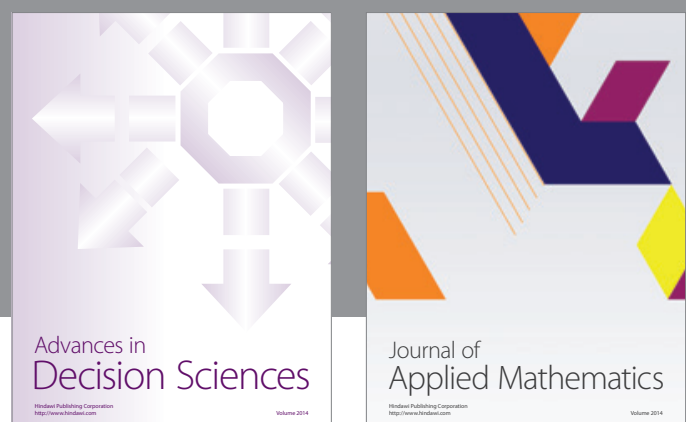

Journal of

Applied Mathematics
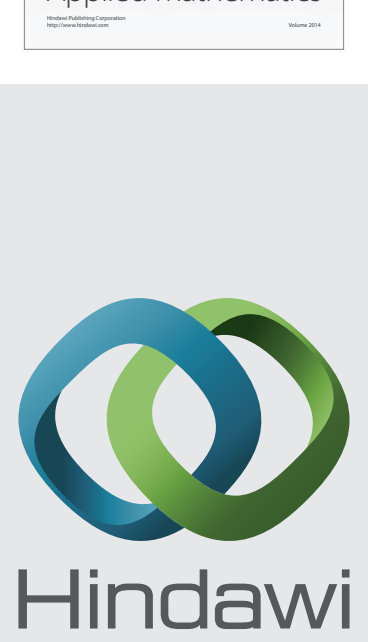

Submit your manuscripts at http://www.hindawi.com
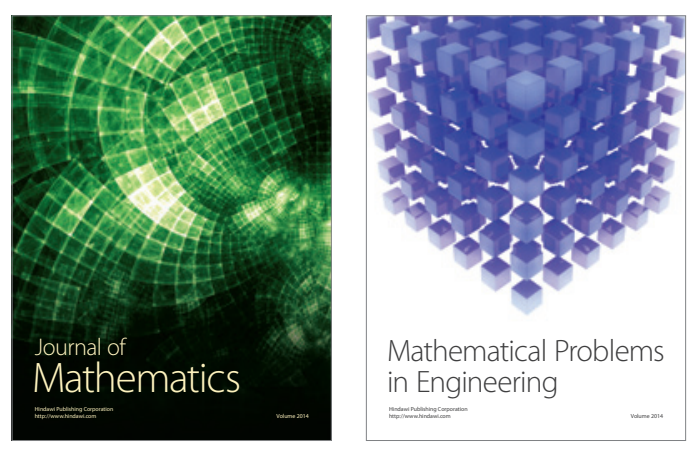

Mathematical Problems in Engineering
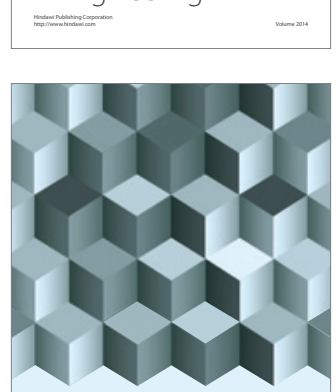

Journal of

Function Spaces
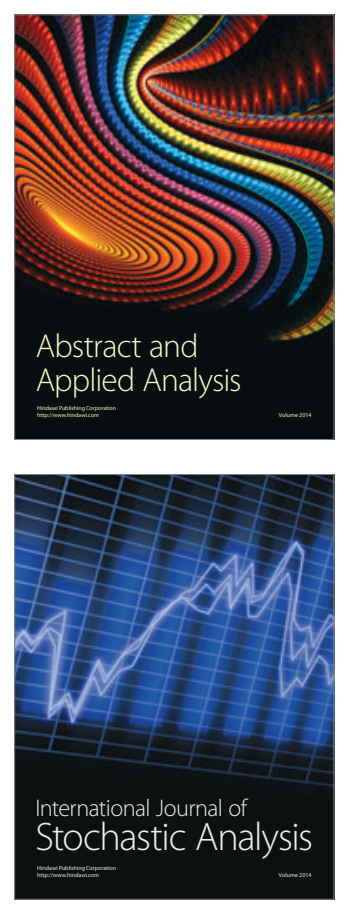

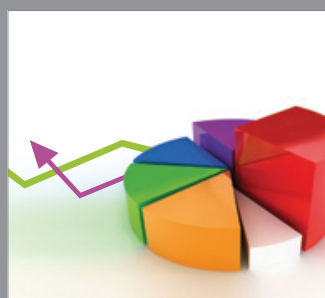

ournal of

Probability and Statistics

Promensencen
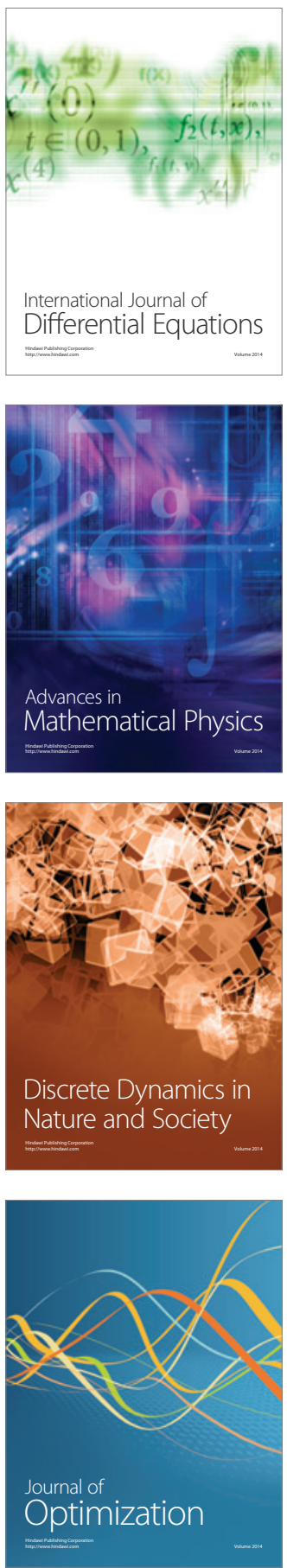